\title{
A Dynamic Algorithm for Reachability Games Played on Trees
}

\author{
Bakhadyr Khoussainov ${ }^{1}$, Jiamou Liu ${ }^{2}$, Imran Khaliq ${ }^{2}$ \\ Department of Computer Science, University of Auckland, New Zealand \\ ${ }^{1}$ bmk@cs . auckland.ac.nz \\ ${ }^{2}\{$ jliu036, ikha020\}@aucklanduni.ac.nz
}

\begin{abstract}
Our goal is to start the investigation of dynamic algorithms for solving games that are played on finite graphs. The dynamic game determinacy problem calls for finding efficient algorithms that decide the winner of the game when the underlying graph undergoes repeated modifications. In this paper, we focus on turn-based reachability games. We provide an algorithm that solves the dynamic reachability game problem on trees. The amortized time complexity of our algorithm is $O(\log n)$, where $n$ is the number of nodes in the current graph.
\end{abstract}

\section{Introduction}

We start to investigate dynamic algorithms for solving games that are played on finite graphs. Games played on graphs, with reachability, Büchi, Muller, Streett, parity and similar type of winning conditions, have recently attracted a great attention due to connections with model checking and verification problems, automata and logic [6][11][13][17]. Given one of these games, to solve the game means to design an (efficient) algorithm that tells us from which nodes a given player wins the game. Polynomial time algorithms exist to solve some of these games, while efficient algorithms for other games remain unknown. For example, on a graph with $n$ nodes and $m$ edges, the reachability game problem is in $O(n+m)$ and is PTIME-complete [8], and Büchi games are in $O(n \cdot m)$ [1].

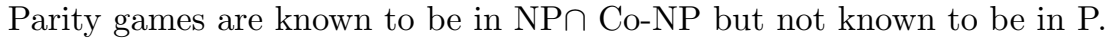

An algorithm for solving the games is static if the games remain unchanged over time. We pose the dynamic game determinacy problem:

We would like to maintain the graph of the game that undergoes a sequence of update and query operations in such a way that facilitates an efficient solution of the current game.

Contrary to the static case, the dynamic determinacy problem takes as input a game $\mathcal{G}$ and a (finite or infinite) sequence $\alpha_{1}, \alpha_{2}, \alpha_{3}, \ldots$ of update or query operations. The goal is to optimize the average running time per operation over a worst-case sequence of operations. This is known as the amortized running time of the operations. 
There has recently been increasing interest in dynamic graph algorithms (See, for example, [4][5]). The dynamic reachability problem on graphs have been investigated in a series of papers by King [9], Demetrescu and Italiano [3], Roditty [14] and Roditty and Zwick [15][16]. In [16], it is shown that for directed graphs with $m$ edges and $n$ nodes, there is a dynamic algorithm for the reachability problem which has an amortized update time of $O(m+n \log n)$ and a worst-case query time of $O(n)$. This paper extends this line of research to dynamic reachability game algorithms. In the setting of games, for a given directed graph $G$ and a player $\sigma$, a set of nodes $T$ is reachable from a node $u$ in $G$ means that there is a strategy for player $\sigma$ such that starting from $u$, all paths produced by player $\sigma$ following that strategy reach $T$, regardless of the actions of the opponent. Hence, the dynamic reachability game problem can be viewed as a generalization of the dynamic reachability problem for graphs.

We now describe two-person reachability games played on directed finite graphs. The two players are Player 0 and Player 1 . The arena $\mathcal{A}$ of the game is a directed graph $\left(V_{0}, V_{1}, E\right)$, where $V_{0}$ is a finite set of 0 -nodes, $V_{1}$ is a finite set of 1-nodes disjoint from $V_{0}$, and $E \subseteq V_{0} \times V_{1} \cup V_{1} \times V_{0}$ is the edge relation. We use $V$ to denote $V_{0} \cup V_{1}$. A reachability game $\mathcal{G}$ is a pair $(\mathcal{A}, T)$ where $\mathcal{A}$ is the arena and $T \subseteq V$ is the set of target nodes for Player 0 .

The players start by placing a token on some initial node $v \in V$ and then move the token in rounds. At each round, the token is moved along an edge by respecting the direction of the edge. If the token is placed at $u \in V_{\sigma}$, where $\sigma \in\{0,1\}$, then Player $\sigma$ moves the token from $u$ to a $v$ such that $(u, v) \in E$. The play stops when the token reaches a node with no out-going edge or a target node. Otherwise, the play continues forever. Formally, a play is a (finite or infinite) sequence $\pi=v_{0} \quad v_{1} v_{2} \ldots$ such that $\left(v_{i}, v_{i+1}\right) \in E$ for all $i$. Player 0 wins the play $\pi$ if $\pi$ is finite and the last node in $\pi$ is in $T$. Otherwise, Player 1 wins the play.

In this paper we provide an algorithm that solves the dynamic reachability game played on trees. We investigate the amortized time complexity of the algorithm. We concentrate on trees because: (1) Trees are simple data structures, and the study of dynamic algorithms on trees is the first step towards the dynamic game determinacy problem. (2) Even in the case of trees the techniques one needs to employ is non-trivial. (3) The amortized time analysis for the dynamic reachability game problem on graphs, in general case, is an interesting hard problem. (4) Finally, we give a satisfactory solution to the problem on trees. We show that the amortized time complexity of our algorithm is of order $O(\log n)$, where $n$ is the number of nodes on the tree. The space complexity of our algorithm is $O(n)$.

\section{A Static Algorithm for Reachability Games}

Let $\mathcal{G}=(\mathcal{A}, T)$ be a reachability game. A (memoryless) strategy for Player $\sigma$ is a partial function $f_{\sigma}: V_{\sigma} \rightarrow V_{1-\sigma}$. A play $\pi=v_{0} v_{1} \ldots$ conforms $f_{\sigma}$ if $v_{i+1}=f_{\sigma}\left(v_{i}\right)$ whenever $v_{i} \in V_{\sigma}$ and $f_{\sigma}$ is defined on $v_{i}$ for all $i$. All strategies in this paper 
are memoryless. A winning strategy for Player $\sigma$ from $v$ is a strategy $f_{\sigma}$ such that Player $\sigma$ wins all plays starting from $v$ that conform $f_{\sigma}$. A node $u$ is a winning position for Player $\sigma$, if Player $\sigma$ has a winning strategy from $u$. The $\sigma$-winning region, denoted $W_{\sigma}$, is the set of all winning positions for Player $\sigma$. Note that the winning regions are defined for memoryless strategies. A game enjoys memoryless determinacy if the regions $W_{0}$ and $W_{1}$ partition $V$.

Theorem 1 (Reachability game determinacy[7]). Reachability games enjoy memoryless determinacy. Moreover, there is an algorithm that computes $W_{0}$ and $W_{1}$ in time $O(n+m)$.

Proof. For $Y \subseteq V$, set $\operatorname{Pre}(Y)=\left\{v \in V_{0} \mid \exists u[(v, u) \in E \wedge u \in Y]\right\} \cup\left\{v \in V_{1} \mid\right.$ $\forall u[(v, u) \in E \rightarrow u \in Y]\}$. Define a sequence $T_{0}, T_{1}, \ldots$ such that $T_{0}=T$, and for $i>0, T_{i}=\operatorname{Pre}\left(T_{i-1}\right) \cup T_{i-1}$. There is an $s$ such that $T_{s}=T_{s+1}$. We say a node $u$ has $\operatorname{rank} r, r \geq 0$, if $u \in T_{r}-T_{r-1}$. A node $u$ has infinite rank if $u \notin T_{s}$. Once checks that a node $u \in W_{0}$ if and only if $u$ has a finite rank. Computing $W_{0}$ takes $O(n+m)$ time.

\section{Dynamic Reachability Game Problem: A Set-Up}

As mentioned above, the dynamic game determinacy problem takes as input a reachability game $\mathcal{G}=(\mathcal{A}, T)$ and a sequence $\alpha_{1}, \alpha_{2}, \ldots$ of update and query operations. The operations produce the sequence of games $\mathcal{G}_{0}, \mathcal{G}_{1}, \ldots$ such that $\mathcal{G}_{i}$ is obtained from $\mathcal{G}_{i-1}$ by applying the operation $\alpha_{i}$. A dynamic algorithm should solve the game $\mathcal{G}_{i}$ for each $i$. We use the notation

$$
\mathcal{A}_{i}=\left(V_{0, i} \cup V_{1, i}, E_{i}\right), V_{i}, T_{i}, W_{\sigma, i}
$$

to denote the arena, the set of nodes, the target set and $\sigma$-winning region for $\mathcal{G}_{i}$. We define the following six update operations and one query operation:

1. InsertNode $(u, i, j)$ operation, where $i, j \in\{0,1\}$, creates a new node $u$ in $V_{i}$. Set $u$ as a target if $j=1$ and not a target if $j=0$.

2. DeleteNode $(u)$ deletes $u \in V$ where $u$ is isolated, i.e., with no incoming or outgoing edge.

3. InsertEdge $(u, v)$ operation inserts an edge from $u$ to $v$.

4. DeleteEdge $(u, v)$ operation deletes the edge from $u$ to $v$.

5. SetTarget $(u)$ operation sets node $u$ as a target.

6. UnsetTarget $(u)$ operation sets node $u$ not as a target.

7. Query $(u)$ operation returns true if $u \in W_{0}$ and false if $u \in W_{1}$

By Theorem 1, each node of $V_{i}$ belongs to either $W_{0, i}$ or $W_{1, i}$.

Definition 1. A node $u$ is in state $\sigma$ if $u \in W_{\sigma}$. The node $u$ changes its state at stage $i+1$, if $u$ is moved either from $W_{0, i}$ to $W_{1, i+1}$ or from $W_{1, i}$ to $W_{0, i+1}$. 
Using the static algorithm from Theorem 1, one produces two lazy dynamic algorithms for reachability games. The first algorithm runs the static algorithm after each update and therefore query takes constant time at each stage. The second algorithm modifies the game graph after each update operation without re-computing the winning positions, but the algorithm runs the static algorithm for the Query(u) operation. In this way, the update operations take constant time, but Query $(u)$ takes the same time as the static algorithm. The amortized time complexity in both algorithms is the same as the static algorithm.

\section{Reachability Game Played on Trees}

This section is the main technical contribution of the paper. All trees are directed, and we assume that the reader is familiar with basic terminology for trees. A forest consists of pairwise disjoint trees. Since the underlying tree of the game undergos changes, the game will in fact be played on forests. We however still say that a reachability game $\mathcal{G}$ is played on trees if its arena is a forest $F$. We describe a fully dynamic algorithm for reachability games played on trees.

A forest $F$ is implemented as a doubly linked list List $(F)$ of nodes. A node $u$ is represented by the tuple $(p(u), \operatorname{pos}(u), \operatorname{tar}(u))$ where $p(u)$ is a pointer to the parent of $u(p(u)=$ null if $u$ is a root $), \operatorname{pos}(u)=\sigma$ if $u \in V_{\sigma}$ and a boolean variable $\operatorname{tar}(u)=$ true iff $u$ is a target.

Our algorithm supports all the operations listed in Section 3. At stage $s$, the algorithm maintains a forest $F_{s}$ obtained from $F_{s-1}$ after performing an operation. We briefly discuss the operations and their implementations:

- Inputs of the update and query operation are given as pointers to their representatives in the linked list $\operatorname{List}\left(F_{s}\right)$.

- The operations InsertNode and DeleteNode are performed in constant time. The InsertNode $(u, i, j)$ operation links the last node in $\operatorname{List}\left(F_{s}\right)$ to a new node $u$. The DeleteNode $(u)$ operation deletes $u$ from $\operatorname{List}\left(F_{s}\right)$.

- All other update operations and the query operation have amortized time $O(\log n)$, where $n$ is the number of nodes in $V$.

- The InsertEdge $(u, v)$ operation is performed only when $v$ is the root of a tree not containing $u$. InsertEdge $(u, v)$ links the trees containing $u$ and $v$. DeleteEdge $(u, v)$ does the opposite by splitting the tree containing $u$ and $v$ into two trees. One contains $u$ and the other has $v$ as its root.

\subsection{Splay Trees}

We describe splay trees (see [10] for details) which we will use in our algorithm. The splay trees form a dynamic data structure for maintaining elements drawn from a totally ordered domain $D$. Elements in $D$ are arranged in a collection $P_{D}$ of splay trees, each of which is identified by the root element.

- $\operatorname{Splay}(A, u)$ : Reorganize the splay tree $A$ so that $u$ is at the root if $u \in A$. 
- $\operatorname{Join}(A, B):$ Join two splay trees $A, B \in P_{D}$, where each element in $A$ is less than each element in $B$, into one tree.

- $\operatorname{Split}(A, u)$ : Split the splay tree $A \in P_{D}$ into two new splay trees

$$
\operatorname{Above}(u)=\{x \in A \mid x>u\} \text { and } \operatorname{Below}(u)=\{x \in A \mid x \leq u\} .
$$

- $\operatorname{Max}(A) / \operatorname{Min}(A):$ Returns the Max/Min element in $A \in P_{D}$.

Theorem 2 (splay trees[12]). For the splay trees on $P_{D}$, the amortized time of the operations above is $O(\log n)$, where $n$ is the cardinality of $D$.

\subsection{Dynamic Path Partition}

Recall that a node $u$ is in state $\sigma$ at stage $s$ if $u \in W_{\sigma, s}$. Denote the state of $u$ at stage $s$ by $\operatorname{State}_{s}(u)$. The update operations may change the state of $u$. This change may trigger a series of state changes on the ancestors of $u$. The state of $u$ does not change if no update operation is applied to a descendant of $u$. We need to have a ChangeState $(u)$ algorithm which carries out the necessary updates when the state of $u$ is changed. The ChangeState( $u$ ) algorithm will not be executed alone, but will rather be a subroutine of other update operations.

One may propose a naive ChangeState $(u)$ algorithm as follows. We hold for each node $v$ its current state. When ChangeState $(u)$ is called, it first changes the state of $u$, then checks if the parent $p(u)$ of $u$ needs to change its state. If so, we changes the state of $p(u)$, and checks if the parent of $p(u)$ needs to change its state, etc. This algorithm takes $O(n)$ amortized time. Our goal is to improve this time bound. For this, we introduce dynamic path partition explained below.

Let $x<_{F} y$ denote the fact that $x$ is an ancestor of $y$ in forest $F$. Set $\operatorname{Path}(x, y)=\left\{z \mid x \leq_{F} z \leq_{F} y\right\}$.

Definition 2. A path partition of a forest $F$ is a collection $P_{F}$ of sets such that $P_{F}$ partitions the set of nodes in $F$ and each set in $P_{F}$ is of the form Path $(x, y)$ for some $x, y \in F$.

Nodes in Path $(x, y)$ are linearly ordered by $<_{F}$. Call the element of $P_{F}$ that contains $u$ the block of $u$. A block is homogeneous if all its elements have the same state. A path partition $P_{F}$ is homogeneous if each block in $P_{F}$ is homogeneous. For any $u$ in $F_{s}$, set $\nu_{s}(u)=\left|\left\{v \mid(u, v) \in E_{s} \wedge \operatorname{State}_{s}(u)=\operatorname{State}_{s}(v)\right\}\right|$.

Definition 3. A node $u$ in $F_{s}$ is stable at stage $s$ if $u \in T_{s}$ or for some $\sigma \in$ $\{0,1\}, u \in V_{\sigma, s} \cap W_{\sigma, s}$ and $\nu_{s}(u) \geq 2$. We use $Z_{s}$ to denote the set of stable nodes at stage $s$.

The following lemma shows how state changes influence non-stable nodes in homogeneous fragments of blocks. The proof follows from the definitions.

Lemma 1. Suppose $x$ is stable, $x \leq_{F_{s}} y$, Path $(x, y)$ is homogeneous and there is no stable node in $\left\{z \mid x<_{F_{s}} z \leq_{F_{s}} y\right\}$. If $y$ changes state at stage $s+1$ then the nodes in the set $\left\{z \mid x<_{F_{s}} z \leq_{F_{s}} y\right\}$ are precisely those nodes that need to change their states. 
Definition 4. A path partition of $F$ is stable if whenever a node $u$ is stable, it is the $\leq_{F}$-maximum element in its own block.

From this definition, for a stable path partition, if $u$ is stable, all elements $x \neq u$ in the block of $u$ are not stable. An example of a stable and homogeneous partition is the trivial partition consisting of singletons.

At stage $s$, the algorithm maintains two data structures, one is the linked list $\operatorname{List}\left(F_{s}\right)$ as described above, and the other is the path partition $P_{F_{s}}$. Each node $u$ in List $\left(F_{s}\right)$ has an extra pointer to its representative in $P_{F_{s}}$. The path partition is maintained to be homogeneous and stable. Denote the block of $u$ at stage $s$ by $B_{s}(u)$. To obtain logarithmic amortized time, each $B_{s}(u)$ is represented by a splay tree.

\subsection{ChangeState (u) Algorithm}

The ChangeState $(u)$ algorithm carries out two tasks. One is that it changes the states of all the necessary nodes of the underlying forest once $u$ changes its state. The second is that it changes the path partition by preserving its homogeneity and stability properties. For the ease of notations, in this subsection we do not use the subscripts $s$ for the forest $F_{s}$ and the target set $T_{s}$. We write $F$ for $F_{s}$ and $T$ for $T_{s}$.

We explain our ChangeState(u) algorithm informally. Suppose $u$ changes its state at stage $s+1$. The algorithm defines a sequence of nodes $x_{1}>_{F} x_{2}>_{F}$ $\ldots$ where $x_{1}=u$. For $i \geq 1$, the algorithm splits $B_{s}\left(x_{i}\right)$ into two disjoint sets Above $\left(x_{i}\right)$ and Below $\left(x_{i}\right)$ and temporarily sets $B_{s+1}\left(x_{i}\right)=\operatorname{Below}\left(x_{i}\right)$. By homogeneity, all nodes in Below $\left(x_{i}\right)$ have the same state at stage $s$. Change the state of all nodes in $B_{s+1}\left(x_{i}\right)$ (this can be done by Lemma 1) and join the current two blocks containing $u$ and $B_{s+1}\left(x_{i}\right)$ into one. If $\min \left\{B_{s+1}\left(x_{i}\right)\right\}$ is the root, stop the process. Otherwise, consider the parent of $\min \left\{B_{s+1}\left(x_{i}\right)\right\}$ which is $w_{i}=p\left(\min \left\{B_{s+1}\left(x_{i}\right)\right\}\right)$. If $\operatorname{State}_{s}\left(w_{i}\right) \neq \operatorname{State}_{s}\left(x_{i}\right)$ or $w_{i} \in Z_{s}$, stop the loop, do not define $x_{i+1}$ and $B_{s+1}(u)$ is now determined. Otherwise, set $x_{i+1}=w_{i}$ and repeat the above process for $x_{i+1}$. Consider the last $w_{i}$ in the process described above that did not go into the block of $u$. If $w_{i} \notin Z_{s}$ and it becomes stable after the change, split $B_{s}\left(w_{i}\right)$ into $\operatorname{Above}\left(w_{i}\right)$ and $\operatorname{Below}\left(w_{i}\right)$ and declare that $w_{i} \in Z_{s+1}$. These all determine the new partition at stage $s+1$.

Algorithm 1 implements the ChangeState( $u$ ) procedure. The current block of $v$ is denoted by $B(v)$. Elements of $B(v)$ are stored in a splay tree with order $\leq_{F}$. With the root of each splay tree $B(v)$ the variable $q(B(v)) \in\{0,1\}$ is associated to denote the current state of nodes in $B(v)$. The while loop computes the sequence $x_{1}, x_{2}, \ldots$ and $w_{1}, w_{2}, \ldots$ described above using the variables $x$ and $w$. The boolean variable ChangeNext decides if the while loop is active. The boolean variable $\operatorname{Stable}(v)$ indicates whether $v$ is stable. With each stable node $v$, the variable $n(v)$ equals $\nu(v)$ at the given stage.

The next two lemmas imply that the path partition obtained after the execution of ChangeState(u) remains homogeneous and stable. 

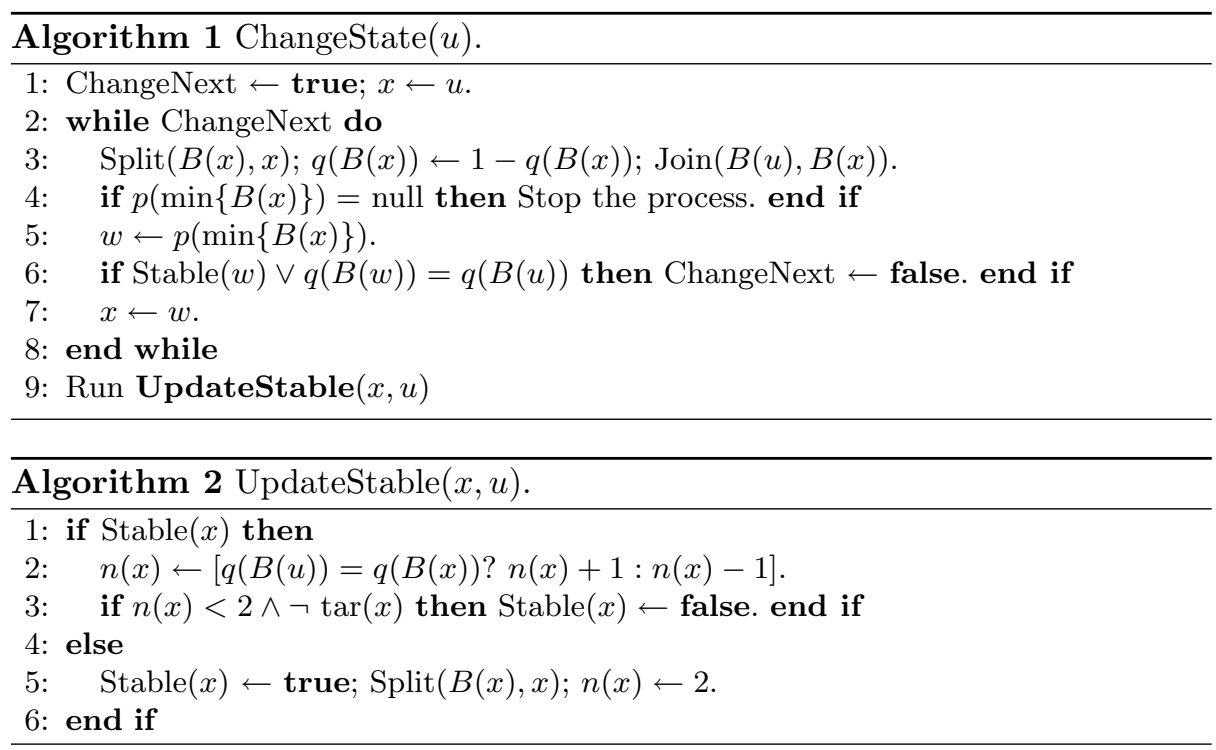

Lemma 2. $B_{s+1}(u)=\left\{z \mid\right.$ State $_{s}(z) \neq$ State $\left._{s+1}(z)\right\}$.

Proof. If $z=u$ then $\operatorname{State}_{s}(z) \neq \operatorname{State}_{s+1}(z)$. Assume $z \neq u$ and $z \in B_{s+1}(u)$. Let $x$ be the $\leq_{F}$-maximum node in $B_{s}(z)$. By definition of $B_{s+1}(u), x \notin Z_{s}$ and $\operatorname{State}_{s}(x)=\operatorname{State}_{s}(u)$. Therefore by Lemma 1 and the construction of the algorithm, $B_{s+1}(u) \subseteq\left\{z \mid \operatorname{State}_{s}(z) \neq \operatorname{State}_{s+1}(z)\right\}$.

Conversely, if $\operatorname{State}_{s}(z) \neq \operatorname{State}_{s+1}(z), z$ must be an ancestor of $u$. Let $x$ be the $\leq_{F}$-minimum node in $B_{s+1}(u)$. If $x$ is the root, $\left\{z \mid \operatorname{State}_{s}(z) \neq\right.$ $\left.\operatorname{State}_{s+1}(z)\right\} \subseteq B_{s+1}(u)$. If $x$ is not the root, either $p(x) \in Z_{s}$ or $\operatorname{State}_{s}(p(x))=$ $\operatorname{State}_{s+1}(u)$. Again by Lemma 1 and description of the algorithm, $\operatorname{State}_{s}(x)=$ $\operatorname{State}_{s+1}(x)$ and thus $\left\{z \mid \operatorname{State}_{s}(z) \neq \operatorname{State}_{s+1}(z)\right\} \subseteq B_{s+1}(u)$.

Lemma 3. Suppose $v \notin Z_{s}$. The node $v \in Z_{s+1}$ if and only if $v$ is the parent of the $\leq_{F}$-least node that changes its state at stage $s+1$.

Proof. Suppose for simplicity that $v \in V_{0, s}$. The case when $v \in V_{1, s}$ can be proved in a similar way. Suppose one of $v$ 's children, say $v_{0}$, is the $\leq_{F}$-least node that changes its state at stage $s+1$. If $\operatorname{State}_{s}(v)=1$, then all of its children are in $W_{1, s}$. Thus $\operatorname{State}_{s+1}\left(v_{0}\right)=0$, and $v$ should also changes to state 0 . This contradicts with the $\leq_{F}$-minimality of $v_{1}$. Therefore $v \in W_{0, s}$. Since $v \notin Z_{s}$, exactly one of its child, say $v_{1}$ is in $W_{0, s}$. If $v_{1}=v_{0}$, then none of $v$ 's children is in $W_{0, s+1}$ and $\operatorname{State}_{s}(v) \neq \operatorname{State}_{s+1}(v)$. Therefore $v_{1} \neq v_{0}$. Thus at stage $s+1$, $v$ has exactly two children in $W_{0, s+1}$ and $v \in Z_{s+1}$.

On the other hand, suppose $v \in Z_{s+1}$. This means that, $v \in W_{0, s+1}$ and $v$ has two children $v_{0}, v_{1}$ in $W_{0, s+1}$. Note that for any $x$, at most one child of $x$ may change state at any given stage. Therefore, at most one of $v_{0}$ and $v_{1}$, say $v_{1}$, is in $W_{0, s}$. Hence $v \in W_{0, s}$, and $\operatorname{State}_{s}(v)=\operatorname{State}_{s+1}(v)$. Therefore $v_{0}$ is the $\leq_{F}$-least node that changes state at stage $s+1$. 


\subsection{Update and Query operations}

We describe the update and query operations. The query operation takes a parameter $u$ and returns $q(B(u)$ ), which is the state variable associated with the root of the splay tree representing $B(u)$. We use an extra variable $c(u)$ to denote the current number of children of $u$.

In principle, the algorithms for operations InsertEdge $(u, v)$, DeleteEdge $(u, v)$, SetTarget $(u)$ and UnsetTarget $(u)$ perform the following three tasks. (1) Firstly, it carries out the update operation on $u$ and $v$. (2) Secondly, it calls ChangeState (u) in the case when $u$ needs to change state. (3) Lastly, it updates $c(z), n(z)$ and Stable $(z)$ for each $z$. Task (1) is straightforward by changing the values of $p(v)$ and $\operatorname{tar}(u)$. Task (2) (3) can be done by using a fixed number of if statements, each with a fixed boolean condition involving comparisons on the variables. We illustrate this using the InsertEdge $(u, v)$ operation as follows.

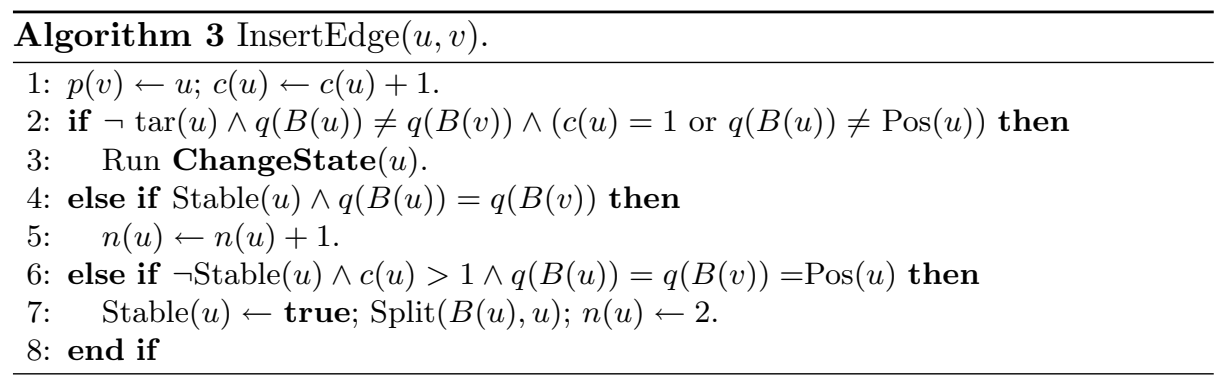

The InsertEdge $(u, v)$ operation is described in Algorithm 3. Suppose the path partition on $F_{s}$ is homogeneous and stable, and

1. $q(B(z))=\operatorname{State}_{s}(z)$.

2. $n(z)=\left|\left\{z^{\prime} \mid\left(z, z^{\prime}\right) \in E_{s} \wedge \operatorname{State}_{s}(z)=\operatorname{State}_{s}\left(z^{\prime}\right)\right\}\right|$.

3. $c(z)=\left|\left\{z^{\prime} \mid\left(z, z^{\prime}\right) \in E_{s}\right\}\right|$.

4. $\operatorname{Stable}(z)$ is true if and only if $z \in Z_{s}$.

Suppose the edge $(u, v)$ is inserted at stage $s+1$. We prove the following two lemmas which imply the correctness of the algorithm.

Lemma 4. ChangeState $(u)$ is called in the $\operatorname{InsertEdge}(u, v)$ algorithm if and only if State $_{s}(u) \neq$ State $_{s+1}(u)$.

Proof. Note that ChangeState $(u)$ is called if and only if the condition for the if statement at Line 2 of Algorithm 3 holds. We prove one direction of the lemma, the other direction is straightforward.

$\operatorname{Suppose} \operatorname{State}_{s}(u) \neq \operatorname{State}_{s+1}(u)$. It must be that $u \notin T_{s}$ and $\operatorname{State}_{s}(u) \neq$ $\operatorname{State}_{s}(v)$. Suppose further that $u$ is not a leaf at stage $s$. If $u \in V_{0, s} \cap W_{0, s}$, there is a child $w$ of $u$ which is also in $W_{0, s}$. This means that $\operatorname{State}_{s}(u)=\operatorname{State}_{s+1}(u)$. Similarly, one may conclude that $u$ does not change state if $u \in V_{1, s} \cap W_{1, s}$. 
Therefore $u \in\left(V_{0, s} \cap W_{1, s}\right) \cup\left(V_{1, s} \cap W_{0, s}\right)$. Therefore the condition for the if statement at Algorithm 3 Line 2 holds.

Lemma 5. Stable $(u)$ is true at stage $s+1$ if and only if $u \in Z_{s+1}$.

Proof. It is easy to see that if $u \in Z_{s}$ then $u \in Z_{s+1}$ and $\operatorname{Stable}(u)$ is set to true at stage $s+1$. Suppose $u \notin Z_{s}$. Note that $\operatorname{Stable}(u)$ is set to true at stage $s+1$ if and only if Line 6 in Algorithm 3 is reached and the condition for the if statement at this line holds, if and only if $u$ is not a leaf at stage $s, \operatorname{State}_{s}(u)=$ $\operatorname{State}_{s+1}(u)=\operatorname{State}_{s}(v)$ and $u \in V_{\sigma, s} \cap W_{\sigma, s}$ for some $\sigma \in\{0,1\}$.

If $u$ is not a leaf, $\operatorname{State}_{s}(u)=\operatorname{State}_{s+1}(u)=\operatorname{State}_{s}(v)$ and $u \in V_{\sigma, s} \cap W_{\sigma, s}$ for some $\sigma \in\{0,1\}$, then there is a child $w$ of $u$ in $W_{\sigma, s}$ and thus $u \in Z_{s+1}$. On the other hand, suppose $u \in Z_{s+1}$. If $u$ changes state, then $u \in V_{\sigma, s} \cap W_{1-\sigma, s}$ for some $\sigma \in\{0,1\}$. This means all children of $u$ are in $W_{1-\sigma, s}$ and $u \notin Z_{s+1}$. Therefore it must be that $u$ did not change state. By definition of a stable node, $u$ is not a leaf, $\operatorname{State}_{s}(u)=\operatorname{State}_{s}(v)$ and $u \in V_{\sigma, s} \cap W_{\sigma, s}$.

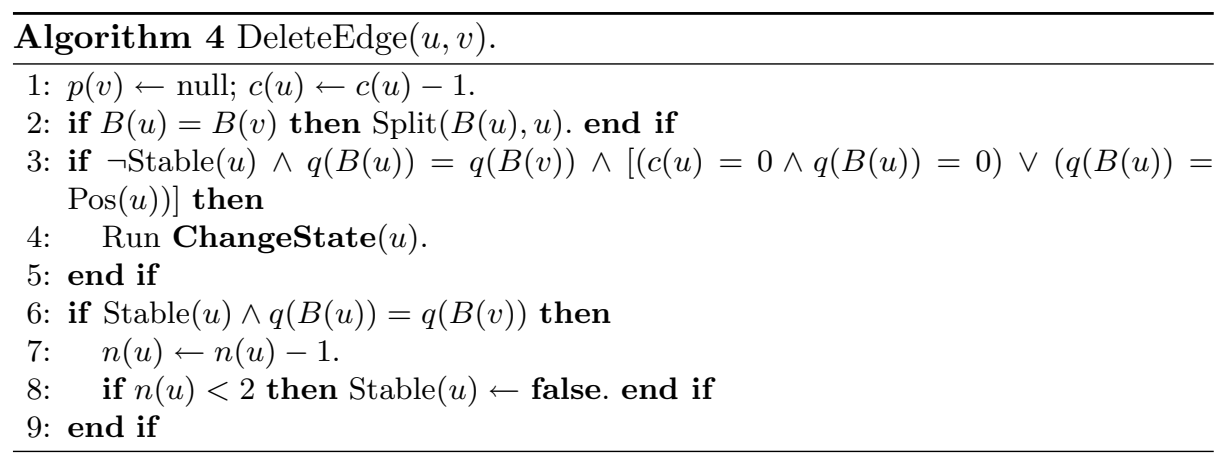

The DeleteEdge $(u, v)$ operation is described in Algorithm 4. Suppose that the edge $(u, v)$ is deleted at stage $s+1$. We also have the following two lemmas which imply the correctness of the algorithm. The proofs are similar in spirit to the proofs of Lemma 4 and Lemma 5.

Lemma 6. ChangeState $(u)$ is called in the DeleteEdge $(u, v)$ algorithm if and

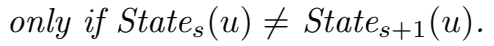

Lemma 7. Stable $(u)$ is true at stage $s+1$ if and only if $u \in Z_{s+1}$.

The SetTarget $(u)$ and UnsetTarget $(u)$ operations are described in Algorithm 5 and Algorithm 6, respectively.

\subsection{Correctness}

The next lemma implies the correctness of the algorithms 1-6. 

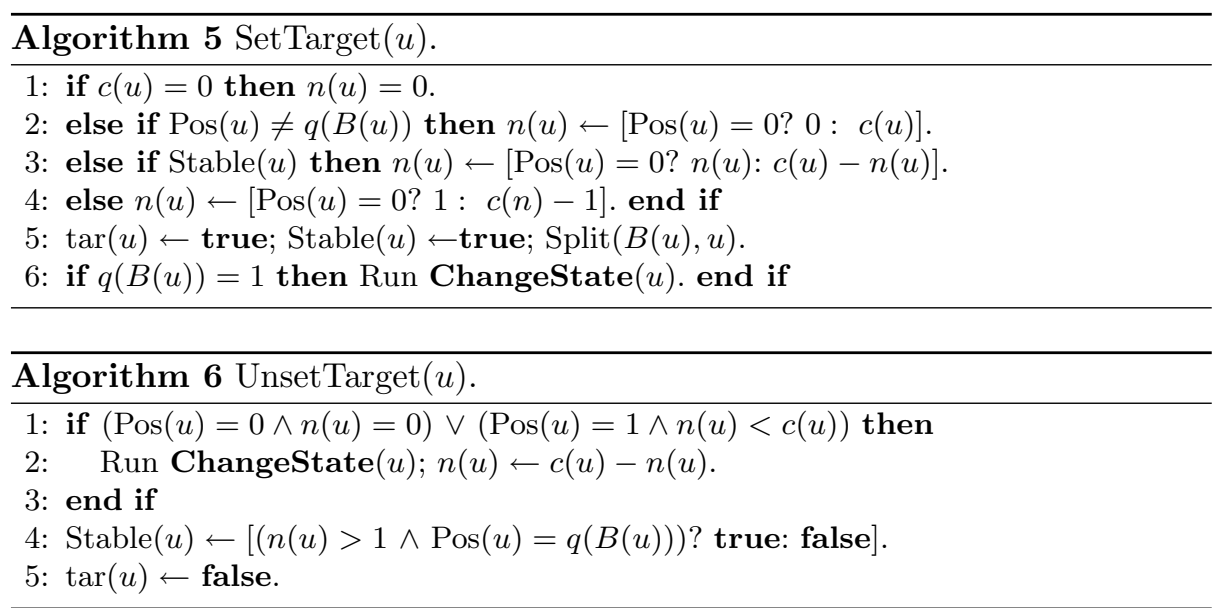

Lemma 8. At each stage $s$, State $_{s}(z)=q(B(z))$ for all node $z \in F_{s}$.

Proof. The proof proceeds by induction on $s$. For simplicity, we assume that the initial forest $F_{0}$ contains only isolated nodes. We set for each node $z, p(z)=$ null, $c(z)=n(z)=0, B(z)=\{z\}$, $\operatorname{Stable}(z)=$ true if and only if $q(B(z))=0$ if and only if $\operatorname{tar}(z)=$ true. For the case when $F_{0}$ is an arbitrary forest, we may assume that the variables $c(z), n(z), B(z)$, Stable $(z)$ and $q(B(z))$ has been pre-set to their respect values as described in the previous subsection. We use $\gamma_{s}(u)$ to denote the number of children of $u$ in stage $s$ and recall that $\nu_{s}(u)$ is the number of $u$ 's children in the same state as $u$. The lemma follows from the fact that the following six inductive assumptions are preserved at each stage $s$.

(1) The set $\left\{B(v) \mid v \in V_{0, s} \cup V_{1, s}\right\}$ forms a homogeneous path partition of $F_{s}$.

(2) For each $z, c(z)=\gamma_{s}(z)$.

(3) For each $z, n(z)=\nu_{s}(z)$ whenever $z$ is stable.

(4) For each $z, \operatorname{Stable}(z)$ is set to True if and only if $z$ is a stable node.

(5) The path partition $\{B(v) \mid v \in V\}$ is stable.

(6) For each $z$, State $_{s}(z)=q(B(z))$.

\section{Complexity}

We analyze the amortized complexity of our algorithm. Each InsertEdge $(u, v)$, DeleteEdge $(u, v)$, SetTarget( $u)$, and UnsetTarget( $u)$ algorithm runs at most once the ChangeState $(u)$ algorithm, a fixed number of splay tree operations, and a fixed number of other low-level operations such as pointer manipulations and comparisons. By Theorem 2, each splay tree operation takes amortized time $O(\log n)$ where $n$ is the number of nodes in the forest. Therefore, the amortized time complexity for these operations is $O(\log n)$ plus the amortized time taken by the ChangeState $(u)$ algorithm. 
We now focus on the amortized time complexity of the ChangeState $(u)$ algorithm. The algorithm runs in iterations. At each iteration, it processes the current block of nodes $B(x)$ and examines the parent $w$ of the $\leq_{F}$-least node in $B(x)$. If $w$ does not exist or does not need to change state, the algorithm stops and calls UpdateStable $(w, x)$; otherwise, the algorithm sets $x$ to $w$ and starts another iteration. Each iteration in the algorithm and the UpdateStable $(w, x)$ algorithm both run a fixed number of splay tree operations and therefore takes amortized time $O(\log n)$. Therefore the algorithm takes time $O(\tau \log n+\log n)$ to execute a sequence of $k$ update operations, where $\tau$ is the number of iterations of ChangeState $(u)$. We prove the following lemma which implies the $O(\log n)$ amortized time complexity of the above update operations.

Lemma 9. For any sequence of $k$ update operations, the total number of iterations ran by the ChangeState $(u)$ algorithm is $O(k)$.

Proof. Given a forest $F=(V, E)$ and path partition $P_{F}$. For a node $u \in V$, let $B(u)$ denote the block of $u$. Define $E_{F}^{P} \subseteq P_{F}^{2}$ such that for all $u, v \in V$

$$
(B(u), B(v)) \in E_{F}^{P} \text { if and only if }(\exists w \in B(u))\left(\exists w^{\prime} \in B(v)\right)\left(w, w^{\prime}\right) \in E
$$

The pair $\left(P_{F}, E_{F}^{P}\right)$ also forms a forest, which we call the partition forest of $P_{F}$.

We prove the lemma using the accounting method (see [2]). At each stage $s$, we define the credit function $\rho_{s}: P_{F_{s}} \rightarrow \mathbb{N}$. For block $B \in P_{F_{0}}$, let $\rho_{0}(B)$ be the number of children of $B$ in $P_{F_{0}}$. At each stage $s+1$, the credit function $\rho_{s+1}$ is obtained from $\rho_{s}$ with the following requirements:

- We create for each execution of $\operatorname{InsertEdge}(u, v)$ and ChangeState(u) an amortized cost of 1 . This cost contributes towards the credit $\rho_{s}(B(u))$. Note that we can create amortized cost at most 2 at each stage.

- For each iteration of ChangeState $(u)$, we take out 1 from $\rho_{s}(B)$ of some $B \in P_{F_{s}}$.

Let $t_{s}$ be the total number of iterations ran at stage $s$. Our goal is to define $\rho_{s}$ in such a way that for any $s>0$,

$$
\sum_{B \in P_{F_{s}}} \rho_{s}(B) \leq \sum_{B \in P_{F_{s-1}}} \rho_{s-1}(B)+2-t_{s}
$$

and $\rho_{s}(B) \geq 0$ for any $B \in P_{F_{s}}$. Note that the existence of such a credit function $\rho_{s}$ implies for any $k$,

$$
\sum_{s=1}^{k} t_{s} \leq 2 k \in O(k) .
$$

We define our desired credit function $\rho_{s}$ by preserving, for every stage $s$, the following invariant:

$$
\left(\forall B \in P_{F_{s}}\right) \quad \rho_{s}(B)=\left|\left\{B^{\prime} \in P_{F_{s}} \mid\left(B, B^{\prime}\right) \in E_{F_{s}}^{P}\right\}\right| .
$$

The detailed procedure for defining $\rho_{s}$ is omitted due to space restriction. 
Theorem 3. There exists a fully dynamic algorithm to solve reachability games played on trees which supports InsertNode $(u, i, j)$ and $\operatorname{DeleteNode}(u)$ in constant time, and InsertEdge $(u, v)$, DeleteEdge $(u, v)$, SetTarget $(u)$, UnsetTarget $(u)$ and Query $(u)$ in amortized $O(\log n)$-time where $n$ is the number of nodes in the forest. The space complexity of the algorithm is $O(n)$.

Proof. By Lemma 9, the update operations have amortized time complexity $O(\log n)$. The splay tree data structures take space $O(n)$ at each stage.

\section{References}

1. Chatterjee, K., Henzinger, T., Piterman, N.: Algorithms for büchi games. In: Proceedings of the 3rd Workshop of Games in Design and Verification (GDV'06). (2006)

2. Cormen, T., Leiserson, C., Rivest, R., Stein, C.: Introduction to algorithms, Second Edition. MIT Press and McGraw-Hill. (2001)

3. Demetrescu, C., and Italiano, G.: Fully dynamic transitive closure: Breaking through the $O\left(n^{2}\right)$ barrier. In: Proceedings of FOCS'00, pp. 381-389. (2000)

4. Demetrescu, C., and Italiano, G.: A new approach to dynamic all pairs shortest paths. In: Proceedings of STOC'03, pp. 159-166. (2003)

5. Eppstein, D., Galil, Z., Italiano, G.: Dynamic graph algorithms. In: Algorithms and Theoretical Computing Handbook, M. Atallah, ed., CRC Press. (1999)

6. Grädel, E.: Model checking games. In: Proceedings of WOLLIC'02, vol.67 of Electronic Notes in Theoretical Computer Science. Elsevier. (2002)

7. Grädel, E., Thomas, W., Wilke, T.: Automata, logics, and infinite games. LNCS 2500, Springer, Heidelberg. (2002)

8. Immerman, N.: Number of quantifiers is better than number of tape cells. In: Journal of Computer and System Sciences, 22:384-406. (1981)

9. King, V.: Fully dynamic algorithms for maintaining all-pairs shortest paths and transitive closure in digraphs. In: Proceedings of FOCS'99, pp 81-91. (1999)

10. Kozen, D.: The design and analysis of algorithms(Monographs in computer science), Springer-Verlag, New York. (1992)

11. Murawski, A.: Reachability games and game semantics: Comparing nondeterministic programs. In: Proceedings of LICS'08, pp. 353-363. (2008)

12. Tarjan, R.: Data structures and network algorithms, vol 44 of Regional Conference Series in Applied Mathematics. SIAM (1983)

13. Radmacher, F., Thomas, W.: A game theoretic approach to the analysis of dynamic networks. In: Proceedings of the 1st Workshop on Verification of Adaptive Systems, VerAS 2007, vol 200(2) of Electronic Notes in Theoretical Computer Science, pp. 21-37. Kaiserslautern, Germany (2007)

14. Rodity, L.: A faster and simpler fully dynamic transitive closure. In: Proceedings of SODA'03, pp. 401-412. (2003)

15. Roditty, L., Zwick, U.: Improved dynamic reachability algorithm for directed graphs. In: Proceedings of FOCS'02, pp. 679-689. (2002)

16. Roditty, L., Zwick, U.: A fully dynamic reachability algorithm for directed graphs with an almost linear update time. In: Proceedings of 36th ACM Symposium on Theory of Computing (STOC'04), pp. 184-191. (2004)

17. Thomas, W.: Infinite games and verification. In: Proceedings of the International Conference on Computer Aided Verification CAV'02, LNCS 2404:58-64. (2002) 